\title{
Reflexiones emergentes de prácticas de un grupo colaborativo de profesores sobre los conocimientos necesarios para enseñar Matemática
}

\author{
José Fernandes da Silva \\ jose.fernandes@ifmg.edu.br \\ https://orcid.org/0000-0002-5798-5379 \\ Instituto Federal de Educação, Ciência e Tecnologia de Minas Gerais - IFMG \\ Guanhães, Brasil. \\ Ana Lúcia Manrique \\ analuciamanrique@gmail.com \\ https://orcid.org/0000-0002-7642-0381 \\ Pontifícia Universidade Católica de São Paulo-PUC/SP \\ São Paulo, Brasil.
}

Recibido: 01/11/2020 Aceptado: 01/12/2020

\begin{abstract}
Resumen
Este artículo tiene como objetivo presentar reflexiones sobre prácticas de profesores de Matemáticas, egresados de una universidad nacional brasileña, sobre los conocimientos necesarios para planificar, desarrollar y evaluar las clases. Para ello, se creó un grupo colaborativo con cuatro profesores voluntarios, que enseñan en los diferentes niveles y modalidades. Como criterio, para participar del grupo colaborativo, el profesor necesitaba haber tenido experiencias de iniciación en la docencia en su formación inicial. Es una investigación cualitativa que se valió de las siguientes estratagemas para recoger datos: aplicación de cuestionarios, observaciones, videos, planificación y reflexión de clases. Los docentes presentan aspectos de las facetas epistémicas y ecológicas, especialmente cuando en sus prácticas de planificación, implementación y reflexión muestran una base matemática sólida y la capacidad de promover el diálogo entre los contenidos y el contexto social, económico y cultural de los estudiantes.
\end{abstract}

Palabras clave: Formación del Profesorado. Enseñanza de las Matemáticas. Conocimiento del Profesor

\section{REFLEXÕES EMERGENTES SOBRE PRÁTICAS DE UM GRUPO COLABORATIVO DE PROFESSORES SOBRE OS CONHECIMENTOS NECESSÁRIOS PARA ENSINAR MATEMÁTICA}

\section{Resumo}

Este artigo tem como objetivo apresentar reflexões sobre as práticas de professores de Matemática, egressos de uma instituição brasileira, sobre os conhecimentos necessários para planejar, desenvolver e avaliar aulas. Para isso, foi criado um grupo colaborativo com quatro professores voluntários, que ensinam em diferentes níveis e modalidades da educação básica. Como critério, para participar do grupo colaborativo, o professor precisava ter experiências de iniciação à docência em sua formação inicial. Trata-se de uma investigação qualitativa que utilizou os seguintes recursos para coleta de dados: aplicação de questionários, observações, 
vídeos, planejamento e reflexão de classe. Os professores apresentam aspectos das facetas epistêmicas e ecológicas, especialmente, quando em suas práticas de planejamento, implementação e reflexão mostram uma sólida base matemática e a capacidade de promover o diálogo entre o conteúdo e o contexto social, econômico e cultural dos alunos.

Palavras chave: Formação de Professores. Ensino de Matemática. Conhecimento do Professor

\title{
Emerging reflections of collaborative group practices on the knowledge needed by the mathematics teacher
}

\begin{abstract}
This article aims to present reflections on the practices of Mathematics teachers, graduates from a Brazilian national university, on the kinds of knowledge required in order to plan, develop and evaluate classes. For this purpose, a collaborative group was created with four volunteer teachers, who teach at different levels and modalities. As a criterion, to participate in the collaborative group, teachers needed to have had experiences of initiation to teaching in their initial training. It is a qualitative research work that made use of the following resources for collecting data: application of questionnaires, observations, videos, planning and reflection on classes. Teachers present aspects of the epistemic and ecological planes. Especially when in their planning, implementation and reflection practices they show a solid mathematical base and the ability to promote dialogue between content and the social, economic and cultural context of the students.
\end{abstract}

Keywords: Teacher Education. Mathematics Education. Teacher Knowledge

\section{Introducción}

Existen en la actualidad un conjunto importante de investigaciones que reportan a los conocimientos que los docentes deben poseer para enseñar los contenidos propuestos por los currículos, en especial, el profesor de Matemática. Se sabe que al entrar en el aula a este profesional le es requerido poner en juego sus conocimientos de modo que favorezca el aprendizaje de los alumnos. Asimismo, es un hecho que el aula está inmersa en un conjunto de factores sociales, culturales, políticos y económicos que influyen en el proceso de enseñanza y aprendizaje (Ricoy, Couto, 2018).

Una de las justificaciones para la proposición de esta investigación deriva del contexto del Programa Institucional de Becas de Iniciación en la Docencia - PIBID. Esta política pública fue creada por la Resolución Normativa No 38, del 12 de diciembre de 2007, del Ministerio de Educación (MEC/Brasil) bajo responsabilidad de la Coordinación de Perfeccionamiento de Personal de Nivel Superior - Capes, y adquirió un espacio importante en el contexto de la formación inicial de profesores brasileños. 
Las instituciones de enseñanza superior postulan proyectos al PIBID y aquellos que son aprobados reciben becas de iniciación en la docencia para los alumnos de diversos cursos de licenciatura. De esa forma, uno de los objetivos del PIBID es anticipar el vínculo entre los futuros profesores y las aulas de la red pública de enseñanza. En esta visión, el PIBID posibilita articular los cursos de formación inicial de profesores con la escuela y con los sistemas de enseñanza de los estados y municipios brasileños.

Muchas investigaciones han sido realizadas en el sentido de identificar repercusiones del PIBID en la formación inicial de los profesores de Matemática (Correia, 2012; Amâncio, 2012; Silva, 2014; Tinti, 2012; Benites, 2013; Largo, 2013; Corrêa, 2013; Moura, 2013; Ribeiro, 2012; Brasil, 2014; Vieira, 2014; Neves, 2014; Schaefer, 2015; Canteiro, 2015; Barros, 2016; Rodrigues, 2016; Vicente, 2016; Carvalho, 2016; Abreu, 2016;Carvalho, 2016;Mendonça, 2016; Tinti y Manrique, 2019; Tinti y Manrique, 2016). Sin embargo, es menester ahondar las reflexiones sobre prácticas profesionales de profesores que participaron de este programa a lo largo de su formación y que en la actualidad están dictando clases en la Educación Básica (Silva, 2014; Zaqueu, 2014).

Corrobora, asimismo, como motivación para esta investigación, la realización de discusiones y ahondamientos teóricos sobre los conocimientos de los profesores que enseñan Matemática. Es un hecho que, desde los estudios de Shulman (1986; 1987), diferentes grupos vienen ampliando las discusiones y, consecuentemente, las bases de conocimientos para la docencia en Matemática vienen ampliándose significativamente. Como ejemplos de estas ampliaciones teóricas, podemos citar el abordaje del Conocimiento Didáctico-Matemático CDM propuesto por Godino (2009), Pino-Fan, Godino y Font (2013), Pino-Fan y Godino (2015) y Pino-Fan, Assis y Castro (2015).

En tal sentido, este artículo tiene como objetivo central investigar cómo un grupo colaborativo formado por profesores egresados de un Curso de Licenciatura en Matemática, y que fueron participantes del PIBID, movilizan sus conocimientos ante el proceso de enseñanza y aprendizaje de contenidos de Matemática. Para ello, nos sirve como norte la siguiente pregunta principal: ¿Cómo articulan sus conocimientos en el proceso de enseñanza y aprendizaje de contenidos matemáticos profesores que mientras cursaban su licenciatura vivenciaron un proyecto aprobado en el PIBID? 


\section{Marco Teórico}

Hasta la década de 1980, pocos estudios se proponían investigar la formación de profesores. A partir de ese período surgieron importantes modelos, buscando caracterizar el conjunto de conocimientos necesarios para el desarrollo de las prácticas pedagógicas. Los trabajos de Shulman (1986), Grossman (1990), Mishra y Koehler (2006) y Hill, Ball y Schilling (2008) son ejemplos de discusiones que buscaron establecer categorías que constituyen el conocimiento del profesor.

Otros debates también se encargaron de discutir los conocimientos para la docencia, aunque con enfoque en la Matemática, como, por ejemplo: Ball, Lubienski y Mewborn (2001), Rowland, Huckstep y Thwaites (2005), Llinares y Krainer (2006), Ponte y Chapman (2006), Ball, Thames y Phelps (2008), Hill, Ball y Schilling (2008) y Schoenfeld y Kilpatrick (2008).

Ante lo anteriormente citado, reportando a los aportes de la Psicología, de la Matemática, de la Epistemología, de la Pedagogía, de la Sociología, de la Semiótica y de la Didáctica de la Matemática, Godino (2009) defiende que el uso del término "conocimiento didácticomatemático del profesor - CDM" es más adecuado para referirse a la complejidad de conocimientos profesionales de profesores de Matemática (Pino-Fan, Font y Godino, 2014).

Actualmente, las discusiones sobre los conocimientos de los profesores de Matemática han buscado centrarse sobre aspectos más específicos que puedan ser empleados para mejorías continuas en el proceso de enseñanza y aprendizaje (Godino, 2009; Pino-Fan, Font y Godino, 2013; Pino-Fan y Godino, 2015).

El término CDM deviene del contexto del "Enfoque Ontosemiótico del conocimiento y de la instrucción de la Matemática" (EOS) (Godino, Contreras y Font, 2006; Godino, Bencomo, Font y Wilhelmi, 2006). Para Godino, Batanero y Font (2008), el EOS es un marco teórico que tiene como objetivo realizar una articulación entre perspectivas y nociones teóricas diferentes, tratando el conocimiento matemático y su proceso de enseñanza y aprendizaje de forma global.

Como ahondamiento sobre los conocimientos necesarios para el profesor, Godino (2009)

propone un conjunto de facetas que son categorías que organizan y extienden estos conocimientos. La figura 1 ilustra las facetas y los niveles en el ámbito del CDM:

Figura 1. Facetas y niveles del conocimiento didáctico-matemático del 


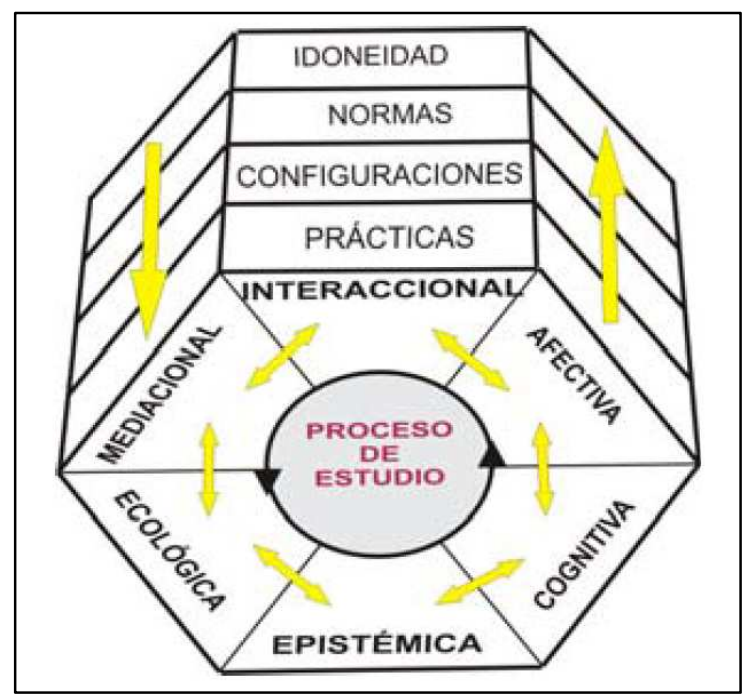

Fuente: Godino (2009)

Las prácticas matemáticas se constituyen en la descripción de las acciones realizadas para resolver las tareas propuestas por el profesor, para contextualizar los contenidos y promover los aprendizajes requeridos (Godino, Font, Wilhelmi y De Castro, 2009). En las configuraciones, los conceptos matemáticos y los procesos didácticos son descritos ante las prácticas con la finalidad de explicitar la complejidad de objetos y significados involucrados en estos procesos. En lo que atañe a las normas y metanormas, se busca la identificación del conjunto de reglas y hábitos que condicionan y tornan posible el proceso de estudio y que afecta a cada faceta y sus interacciones. Por último, la noción de idoneidad didáctica puede auxiliar el análisis de un proceso de enseñanza y aprendizaje, el planeamiento de una clase o, inclusive, de un curso. Asimismo, puede ser útil para analizar materiales didácticos o aspectos específicos de la enseñanza de la Matemática (Godino, 2013; Breda, Font y Lima, 2015).

A continuación, con base en los estudios de Juan Godino y sus colaboradores, presentamos un abordaje más profundado de las facetas que componen el CDM, en especial la Epistémica y la Ecológica en lo que concierne a sus componentes e indicadores.

Cognitiva: esta faceta posibilita que los profesores conozcan mejor a sus alumnos, pues, con la reflexión y la evaluación, es posible, del punto de vista de la institución educativa, acompañar el proceso de aprendizaje.

Afectiva: es la faceta que permite a los profesores lidiar con la parte afectiva, que está comprendida por elementos relacionados con las actitudes, emociones, creencias y valores de los alumnos en el ambiente de estudios de la Matemática. 
Mediacional: se refiere a los conocimientos del profesor relacionados a la capacidad de articular materiales y tecnologías para la enseñanza.

Interaccional: se trata de la capacidad del profesor de comprender, prever, implementar y evaluar las interacciones que ocurren en el proceso de enseñanza y aprendizaje. En este proceso, las relaciones se establecen en contexto: entre profesores y alumnos, alumnos y alumnos, y entre alumnos y los recursos establecidos.

Para la faceta Epistémica y la Ecológica, presentamos los componentes e indicadores de idoneidad, pues ellos serán utilizados en nuestro análisis.

Epistémica: está relacionada con los conocimientos matemáticos involucrados en el contexto educacional y su organización para el proceso de enseñanza. Forman parte de esta faceta los problemas seleccionados, el lenguaje elaborado, los procedimientos, las definiciones y los argumentos utilizados por el profesor.

Cuadro 1. Componentes e indicadores de idoneidad epistémica

\begin{tabular}{c|l}
\hline Componentes & \multicolumn{1}{c}{ Indicadores } \\
\hline $\begin{array}{c}\text { Situaciones - } \\
\text { problemas }\end{array}$ & $\begin{array}{l}\text { Se presenta una muestra representativa y articulada de situaciones de } \\
\text { contextualización, ejercitación y aplicación; Se proponen situaciones } \\
\text { de generación de problemas (problematización) }\end{array}$ \\
\hline Lenguajes & $\begin{array}{l}\text { Uso de diferentes modos de expresión matemática (verbal, gráfica, } \\
\text { simbólica...), traducciones y conversiones entre los mismos; Nivel del } \\
\text { lenguaje adecuado a los niños a que se dirige; Se proponen situaciones } \\
\text { de expresión matemática e interpretación }\end{array}$ \\
\hline Reglas & $\begin{array}{l}\text { Las definiciones y procedimientos son claros y correctos, y están } \\
\text { adaptados al nivel educativo al que se dirigen; Se presentan los } \\
\text { enunciados y procedimientos fundamentales del tema para el nivel } \\
\text { educativo dado; Se proponen situaciones donde los alumnos tengan que } \\
\text { generar o negociar definiciones, proposiciones o procedimientos }\end{array}$ \\
\hline Argumentos & $\begin{array}{l}\text { Las explicaciones, comprobaciones y demostraciones son adecuadas al } \\
\text { nivel educativo a que se dirigen; Se promueven situaciones donde el } \\
\text { alumno tenga que argumentar }\end{array}$ \\
\hline Relaciones & $\begin{array}{l}\text { Los objetos matemáticos (problemas, definiciones, proposiciones, etc.) } \\
\text { se relacionan y conectan entre sí; Se identifican y articulan los diversos } \\
\text { significados de los objetos que intervienen en las prácticas } \\
\text { matemáticas. }\end{array}$ \\
\hline
\end{tabular}

$$
\text { Fuente: Godino (2011, p. 9) }
$$

Ecológica: en el ámbito de esta faceta se espera que el profesor sea capaz de percibir el currículo como una ventana que establece enlaces con el entorno social, político y económico.

Cuadro 2. Componentes e indicadores de idoneidad ecológica

Componentes $\quad$ Indicadores




\begin{tabular}{c|l}
\hline $\begin{array}{c}\text { Adaptación al } \\
\text { currículo }\end{array}$ & $\begin{array}{l}\text { Los contenidos, su implementación y evaluación se corresponden con } \\
\text { las directrices curriculares }\end{array}$ \\
\hline $\begin{array}{c}\text { Apertura hacia la } \\
\text { innovación didáctica }\end{array}$ & $\begin{array}{l}\text { Innovación basada en la investigación y la práctica reflexiva } \\
\text { Integración de nuevas tecnologías (calculadoras, computadoras, TIC, } \\
\text { etc.) en el proyecto educativo }\end{array}$ \\
\hline $\begin{array}{c}\text { Adaptación socio- } \\
\text { profesional y cultural }\end{array}$ & $\begin{array}{l}\text { Los contenidos contribuyen a la formación socio-profesional de los } \\
\text { Estuantes }\end{array}$ \\
\hline $\begin{array}{c}\text { Educación en valores } \\
\text { Conexiones intra e }\end{array}$ & $\begin{array}{l}\text { Se contempla la formación en valores democráticos y el pensamiento } \\
\text { crítico }\end{array}$ \\
\hline Interdisciplinarias & $\begin{array}{l}\text { Los contenidos se relacionan con otros contenidos intra e } \\
\text { interdisciplinarios }\end{array}$ \\
\hline
\end{tabular}

$$
\text { Fuente: Godino (2011, p. 14) }
$$

Así, ante la complejidad del modelo del CDM del profesor, para este artículo, investigamos el nivel de la idoneidad de las facetas epistémica y ecológica.

\section{Metodología}

El abordaje de la pesquisa es cualitativo (Garnica, 2004), en el cual ocurre la legitimidad de no establecer una hipótesis a priori y la no neutralidad del investigador. Tal perspectiva es reforzada por Gatti (2010), cuando señala que un método, en la investigación científica en el área de las ciencias humanas, no es una receta, sino que se trata de una construcción que se realiza en situación.

En cuanto al público destinatario, éste estuvo compuesto por cuatro profesores (P1, P2, P3 y P4), que dictan clases en la Educación Primaria y que, en sus procesos de formación inicial, tuvieron experiencias como becarios de iniciación en la docencia en el PIBID, durante al menos dos años ininterrumpidos y que se ofrecieron como voluntarios para participar de la pesquisa. Los investigadores son designados por mediadores (M1 y M2). La tabla VII explicita el perfil de los participantes:

Cuadro 3. Perfil de los participantes

\begin{tabular}{c|c|c|c|c}
\hline Participante & P1 & P2 & P3 & P4 \\
\hline Edad & 22 & 26 & 22 & 27 \\
\hline $\begin{array}{c}\text { Tiempo de Participación } \\
\text { en el PIBID }\end{array}$ & 3 años & 3 años & 2 años & 3,5 años \\
\hline $\begin{array}{c}\text { Nivel y modalidad en } \\
\text { que dicta clases }\end{array}$ & $\begin{array}{c}\text { Enseñanza } \\
\text { Secundaria }\end{array}$ & $\begin{array}{c}\text { Enseñanza } \\
\text { Primaria y } \\
\text { Secundaria }\end{array}$ & $\begin{array}{c}\text { Enseñanza } \\
\text { Secundaria }\end{array}$ & $\begin{array}{c}\text { Enseñanza } \\
\text { Primaria y } \\
\text { Secundaria }\end{array}$ \\
\hline Localidad & Urbana & Urbana & Urbana & Rural \\
\hline Año de graduación & 2016 & 2016 & 2016 & 2014 \\
\hline
\end{tabular}

Fuente: Elaboración propia 
De lo cuadro presentado es posible inferir que se trata de jóvenes profesores que dictan clases en los diferentes niveles y modalidades de enseñanza de la educación básica.

Con los profesores participantes de la pesquisa se creó un grupo colaborativo en el cual las actividades fueron basadas en la coparticipación de saberes, experiencias y prácticas (Fiorentini, 2004; Palanch y Manrique, 2016).

El grupo se reunía en encuentros semanales en las dependencias de la universidad en que se graduaron, y cada encuentro era dirigido al diálogo y la comunicación entre los participantes, con vistas al conocimiento mutuo y el relato de experiencias sobre sus trayectorias académicas y profesionales, así como la socialización de propuestas de clases. Los encuentros fueron realizados a lo largo del segundo semestre de 2018, totalizando 12 encuentros, que fueron grabados en audios y videos.

En cada encuentro el grupo iniciaba los debates sobre los planes de clases presentados por los participantes. Cada profesor presentaba un planeamiento, relacionado a la rutina de sus clases y a los currículos de las escuelas. Después de cada encuentro, los investigadores observaban la clase del profesor en la escuela donde él o ella se desempeñaba. Las clases fueron grabadas en audio y video, así como fueron realizadas anotaciones en la forma de diario de campo. Inicialmente, se aplicó un cuestionario con el propósito de registrar informaciones más específicas en cuanto a la formación inicial de estos cuatro profesores. Fue utilizado el recurso del Google Forms. Los datos fueron analizados teniendo en cuenta las facetas Ecológica y Epistémica, considerando el nivel de idoneidad didáctica y sus respectivos componentes e indicadores.

\section{Resultados y discusiones}

Los profesores, sus vivencias en el PIBID y sus actuaciones profesionales: elementos para la constitución de un trabajo colectivo

Los participantes de esta investigación son profesores que cuentan con 2 a 3,5 años de participación en el ámbito del PIBID y, como mínimo, 2 años de práctica docente en la Educación Primaria o secundaria, de la red de enseñanza pública. Ante esto, se puede afirmar que los docentes poseen experiencia en el campo de actuación. 
Aunque el PIBID sea un programa que apunta a insertar los futuros profesores en aulas, él también se configura por las repercusiones de los participantes como una política pública de incentivo a la investigación sobre la práctica y a la extensión, conforme relata P1:

El PIBID posibilitó muchos aprendizajes en el aula, y principalmente, fuera de ella. Las experiencias compartidas como consecuencia de las participaciones en los encuentros / congresos / seminarios fueron importantes en el sentido de ubicarme en relación a la situación educacional, así como me auxilió en el uso de diferentes estrategias de enseñanza, además de prepararme para lidiar con la inclusión de alumnos con necesidades educativas especiales. (P1, cuestionario, 2018).

Lo expuesto nos conduce a la reflexión sobre la importancia de la formación de profesores de Matemática dialogar con el entorno y con la comunidad de educadores y especialistas (Godino, 2009), pues el intercambio de experiencias debe ser elemento de los procesos formativos. Las vivencias de las prácticas pedagógicas necesitan ser socializadas, pues la complejidad del proceso de enseñanza y aprendizaje de la Matemática (Breda, Font, Lima, 2015) requiere debates sobre planeamiento, implementación y evaluación. Esta perspectiva puede ser ejemplificada por P2:

En el ámbito del PIBID, presenté un relato de experiencia sobre un trabajo comprendiendo la escritura matemática junto con el uso del material dorado. Fue muy enriquecedor poder planear, elaborar, aplicar y divulgar las experiencias vivenciadas a través de este tipo de trabajo. (P2, cuestionario, 2018).

En las experiencias citadas, notamos que los profesores trajeron al grupo, además de sus vivencias en el aula, un repertorio de discusiones importantes acerca de la Educación Matemática, prácticas de iniciación en la investigación (Gatti, Barretto, André y Almeida, 2019) y consciencia de la importancia de la formación matemática y pedagógica del profesor. Además de lo expuesto, todos los profesores señalaron que el ambiente vivido en el PIBID proporcionó debates y prácticas sobre resolución de problemas y uso de tecnologías, tanto en la institución formadora, como en el desarrollo de sus actividades en las escuelas socias. Tales evidencias coadyuvan con una perspectiva de formación que va más allá de la dimensión matemática (PinoFan y Godino, 2015), esto es, una formación con enfoque en los recursos, interacciones, espacios y contextos. 


\section{Evidencias del conocimiento didáctico-matemático de los egresados del PIBID: planeamiento y contextos educacionales.}

Para las discusiones en el ámbito del grupo, P1 eligió la temática "Pirámides", P2 "Nociones de porcentaje y gráficos", P3 "Introducción a logaritmos” y P4 "Introducción al concepto de función". Tales temáticas estaban previstas en los currículos de las escuelas en que actuaban. Siendo así, destacamos el posicionamiento de P4 que trajo al grupo la idea de un plan de clase para abordar el concepto de función con estudiantes de una escuela localizada en la zona rural:

Voy a empezar el contenido de función con los alumnos. Así, voy a iniciar la clase con dos actividades en las cuales relaciono una función del etanol y de la caña de azúcar para intentar mostrar un poco la realidad de los alumnos y que Brasil es uno de los mayores productores de alcohol, justamente debido a las vastas plantaciones de caña de azúcar. Voy a ver si consigo, en el transcurso de la actividad, que ellos cuestionen sobre las condiciones de biocombustibles, ver con ellos cuáles serían las finalidades de utilizar más este tipo de combustible. (P4, videograbación, 2018)

Cuando cuestionado sobre las razones que lo llevaron a acercar el plan de clase a la realidad de los alumnos, este profesor enfatizó:

Yo no puedo introducir un contenido para el alumno, que está en la zona rural, sin valorizar sus conocimientos. Como participante del PIBID, actué en la zona rural y aprendí a gustar y valorizar las prácticas locales. (P4, transcripción de videograbación, 2018).

Este testigo señala la importancia del PIBID como política pública, para la formación de los profesores, que promueve el diálogo con los diferentes contextos educacionales (Godino, 2009), evitando así que las dificultades del inicio de la carrera provoquen desestimulo y abandono (Tinti y Manrique, 2019).

Similarmente, P2, en su planeamiento, demostró una sensible atención a las condiciones sociales, económicas y culturales de sus alumnos. La escuela donde P2 dicta clases es una institución pública que atiende a alumnos de los años finales de la Enseñanza Primaria y Enseñanza Secundaria. La escuela carece de recursos y medios para apoyar la enseñanza de la Matemática. Sobre esta carencia P2 afirma:

Yo necesito, a veces, pedir materiales prestados en otras instituciones, pues la escuela no tiene Laboratorio de Matemática. (P4, transcripción de videograbación, 2018). 
La actitud de P2 demuestra una preocupación con la instrumentalización de la clase y el protagonismo de los estudiantes en su planeamiento, así como la adaptación del proceso de enseñanza y aprendizaje al contexto de la escuela (Breda, Font y Pino-Fan, 2018).

Planeo mis clases teniendo en vista las posibles dudas que los estudiantes pueden plantear. Cuando actuaba en el PIBID veía cómo un buen planeamiento marcaba la diferencia. Busco analizar cuáles contenidos previos ellos necesitarían para aprender el nuevo contenido. Veo cuán importante es esta preocupación, pues de nada serviría planear mis clases sin tener en cuenta mi público destinatario. ( $\mathrm{P} 2$, transcripción de videograbación, 2018).

Esta capacidad de adaptación del currículo a las realidades demuestra una experiencia de los profesores con los contextos educativos obtenida con la participación en el PIBID.

En lo que atañe a la apertura para la innovación didáctica, los profesores demuestran consciencia sobre la importancia de la tecnología para el proceso de enseñanza y aprendizaje. En especial, P1, que dicta clases en una escuela de la red federal con condiciones estructurales privilegiadas, destaca el auxilio de la tecnología para la enseñanza.

Antes voy a trabajar con los alumnos la cuestión de la semejanza de las pirámides, por ejemplo, el área lateral de la pirámide uno nota que está formada por triángulos, y para complementar tengo también el GeoGebra, y después de la clasificación vamos a destacar los elementos: caras laterales, vértices, altura, base. Inicialmente yo escogí traer una pirámide oblicua, para ya del primer instante borrar la idea de que toda pirámide es bonita y perfecta, como tenemos la costumbre de ver. (P1 transcripción de videograbación, 2018).

Las reflexiones del grupo, sobre la propuesta de clase de P1 colaboraron para que él agregara un video ${ }^{1}$ sobre la Pirámide Teotihuacan, situada en México, para contextualizar mejor el contenido. El siguiente trecho ilustra el momento de colaboración entre los profesores:

P1: La intención es que yo no gaste todo el tiempo sólo presentando el contenido, mi intención es dejar, rápidamente, actividades para que los estudiantes comiencen a hacer.

P4: Creo que uno podría trabajar también un poco de la historia de la Matemática y llevar un poco de la historia de esos contenidos.

M1: El P4 habló sobre la historia del contenido, estaba recordando que vi un reportaje sobre las Pirámides de México. ¿Usted cree que un video de 2 minutos cabría en su presentación?

P1: Y estaría bien también, porque todo el mundo ya tiene en la cabeza que pirámides son tan sólo las del Egipto. Por eso ya sería una novedad.

1Pirámide de Teotihuacán: https://www.youtube.com/watch?v=yiWDiLpn8dg 
Creo que un video pequeño es interesante. (transcripción de videograbación, 2018).

La propuesta de clase de P1, inicialmente, era iniciar la explicación sobre el tema pirámides sin antes movilizar la clase. Sin embargo, el grupo tuvo un rol fundamental en el fomento de la innovación de la práctica educativa (Godino, 2013), especialmente, con la inserción del video. Sobre el uso del GeoGebra, P1 señaló que las experiencias con este software formaron parte de su formación inicial, en especial, en las actividades de iniciación en la docencia en el ámbito del PIBID.

En lo que concierne a la adaptación socioprofesional y cultural, la mayoría de los profesores presentan elementos que buscan relacionar la enseñanza de la Matemática con el mundo del trabajo. Fue evidente la preocupación de los profesores por preparar a los estudiantes para el ingreso a la Enseñanza Superior a través del Examen Nacional de la Enseñanza Media ENEM (Prueba brasileña que permite el ingreso a las universidades). Al respecto de lo expuesto P4 afirma:

Los alumnos del tercer año de la Enseñanza Secundaria, una división con diez, no tiene la base de contenido bien construida. Yo estaba enseñando geometría analítica, y ya iba a entrar en estadística, gráficos, etc. Pero mis alumnos presentan mucha dificultad [...] Fue mejor parar y volver a revisar los contenidos, pues de los diez, creo que sólo dos van a presentarse al ENEM. (P4, transcripción de videograbación, 2018).

Como ya dijimos anteriormente, reconocer el contexto en el cual el proceso de enseñanza y aprendizaje tiene lugar es importante, pues de acuerdo con Godino (2013) la Matemática que enseñamos debe ser útil para los ciudadanos y profesionales, siendo así, coherente con sus principios y razón de ser. Tal postura demuestra un proceso de solidaridad en la práctica pedagógica de estos profesores, o sea, demuestra valores que transcienden el acto de enseñar Matemática.

En mis grupos intento mostrar a los alumnos los límites que cada uno debe tener con su actitud. Intento trabajar con ellos el respeto por el prójimo. Busco trabajar la autoconfianza de los alumnos, mostrando que uno siempre es capaz. Basta creer en sí mismo. (P2, transcripción de videograbación, 2018).

También en esa misma dirección, P2 y P4 demuestran aprecio por el fomento de actitudes basadas en la cooperación entre los estudiantes. 
P2: Mira, son estudiantes que dependiendo del día participan mucho de las clases, hay alumnos que infelizmente no fueron bien alfabetizados, hay algunos desmotivados.

M1: ¿Esto le trae preocupación a usted cuando está elaborando su clase?

P4: Con seguridad, de vez en cuando pido que se sienten juntos y hagan las tareas en grupo para ver si uno ayuda al otro. (transcripción de videograbación, 2018).

Podemos notar, con las reflexiones de los profesores, que el contacto de los profesores con la educación básica desde el inicio de la licenciatura contribuye para fomentar la identidad profesional (Ponte, 2013) y la capacidad de relacionar el mundo teórico con el mundo de la práctica.

\section{Evidencias del conocimiento didáctico-matemático de los egresados del PIBID: la elección de los problemas.}

En el segundo momento, las actividades del grupo colaborativo fueron volcadas a la socialización de las propuestas de clases de los profesores teniendo como foco la calidad de la Matemática a ser enseñada (Godino, 2011). En ese sentido, los profesores creen que tareas desafiadoras son importantes para el abordaje de los contenidos, tal como lo ponen de manifiesto las palabras de $\mathrm{P} 1$ :

[...] para trabajar las pirámides traje algunas ilustraciones para los alumnos, para introducir lo que es el concepto, [...] la semejanza entre todas las figuras (imágenes de pirámides), y cuestionando con ellos la relación que una tiene con la otra. Y a partir de eso nosotros llegaremos a un consenso sobre lo que sería una pirámide y cuál sería su definición, siendo importante que los alumnos tengan un conocimiento previo sobre la geometría espacial de posición. Esa ahí (mostrando la siguiente tarea: Un cubo con arista midiendo 6 está dividido en pirámides congruentes de bases cuadradas y con vértices en el centro del cubo. ¿Cuál es el volumen de cada pirámide?) En una pirámide cualquiera trabajaremos con las pirámides regulares, medidas notables como altura y apotema de la base, y los catetos de un triángulo rectángulo. En esa parte yo traje el GeoGebra [...], pues queda más visible para los niños esa cuestión del triángulo que queda dentro de la pirámide. (P1, videograbación, 2018).

Se percibe una preocupación de P1 en cuanto al rigor y la calidad de la Matemática involucrada en su propuesta de clase. Tal hecho nos conduce a reflexionar sobre la importancia de que el profesor sea capaz de elegir una buena tarea movilizando los principales aspectos del contenido (definiciones, conceptos, representaciones, argumentaciones, proposiciones y 
adecuaciones de estos al nivel educativo de los alumnos) y las habilidades a ser desarrolladas por los estudiantes (Godino, 2009). Reforzando esa perspectiva P3 externa:

Yo ya le he explicado a mi grupo la función exponencial y ahora empezaré la logarítmica. Comenzaré con un problema relativo a la función exponencial: "si una planta duplica su tamaño cada un mes durante el período de su vida, sabiendo que la altura inicial es de $1 \mathrm{~cm}$, ¿cuál es la altura esperada al final del quinto mes?" A partir de ese punto, presento un logaritmo, realizando la pregunta "¿En qué tiempo la altura de la planta es igual a $9 \mathrm{~cm}$ ?", pues eso aquí sería calcular un logaritmo de 9 en la base 2. Y, cuando observamos el gráfico, vemos que 9 centímetros está entre 3 y 4 . El resultado da 3,169, yo también quiero trabajar con ellos ese 0,169 de mes. (P3, videograbación, 2018).

Observamos que para P3 la tarea tiene el objetivo de llevar los estudiantes a reflexionar sobre la siguiente expresión: $2^{x}=9$. O sea, cuál número debe ser el exponente de 2 para que el resultado sea 9. A partir de ese punto, de acuerdo con el profesor, la noción de logaritmo sería introducida.

Podemos notar en la reflexión anteriormente mencionada, la búsqueda, por P3, del conocimiento previo del alumno (función exponencial) para dar inicio al tema de los logaritmos. Se trata de un abordaje que recurre a las nociones intuitivas y formales (Godino, 2009) para generalizar la construcción del conocimiento matemático. Ante lo expuesto, es importante afirmar que los profesores presentaron un conocimiento matemático adecuado para la enseñanza

de esta ciencia. Tal hecho está en consonancia con Godino (2011), cuando afirma que "un programa formativo, o un proceso de estudio matemático, tiene mayor idoneidad epistémica en la medida en que los contenidos implementados (o pretendidos) representan bien a los contenidos de referencia" (Godino, 2011, p. 8).

\section{Repercusiones sobre la implementación de la práctica educativa.}

Las propuestas de clases presentadas por los profesores, en el ámbito del grupo, traían importantes aportes originados en la formación inicial, en especial, de sus experiencias en el ámbito del PIBID. Sin embargo, el debate y la reflexión suscitaron cuestionamientos en torno de las tareas y de las prácticas planeadas "constituyendo un proceso promisorio de desarrollo profesional de los profesores participantes" (Ponte, Baptista, Vélez y Costa, 2012, p. 20). El proceso reflexivo es fundamental para la práctica del profesor de Matemática, desde la fase de 
la construcción del diseño de la clase hasta su realización efectiva (Mallart, Font y Malaspina, 2015).

En el ámbito del grupo, el proceso de reflexión culminó en cambios importantes en los planeamientos de las clases. Entre ellas se destaca la inserción del uso de tecnologías y la búsqueda de videos, trabajos en grupos, trabajos de investigación en clase, alteración de secuencias de tareas y otros. Después de implementar, en sus escuelas, la clase debatida en el ámbito del grupo, los profesores retornaron para reflexionar con los pares sus vivencias e impresiones.

¡La idea de plantear el problema en el primer momento de la clase fue lo que me dejó más sorprendida, ¡salió bien! [...] Los alumnos preguntaron "profesora, ¿cuál es el contenido?”. Ellos querían el contenido antes de la presentación del problema porque, para ellos, tener el problema sin que el profesor haya explicado el contenido no es normal. [...] El problema (sobre pirámide) fue bueno e instigó los chicos, después de discutir el problema asistimos vimos el video, en ese momento ellos no conversaron, literalmente, hubo un silencio total, ellos solo prestaron atención al video. (P1, videograbación, 2018).

El trecho anterior ilustra la importancia de las discusiones en el ámbito del grupo. El planeamiento de P1 estaba volcado al desarrollo de una Matemática robusta, aunque en un modelo más rígido "explicar tema", "pasar ejercicios” y "realizar la corrección” de estos. El debate con los pares posibilitó una dinámica que rompió con el carácter lineal de la clase propuesta.

\section{Consideraciones finales}

Destacamos, por las evidencias de los datos, que el desarrollo de los conocimientos de los profesores de Matemática está en estrecha relación con experiencias que van desde una sólida base matemática, hasta el diálogo con el entorno, en especial, con el futuro campo de trabajo. Las prácticas en el ámbito del grupo colaborativo refuerzan la importancia que los profesores dan a los espacios de discusión y reflexión sobre la enseñanza de la Matemática. Tales espacios pueden contribuir para que los profesores en inicio de carrera fortalezcan sus vivencias en el ámbito de la escuela, evitando que se desencanten con la condición de educador (Ponte, Galvão, Trigo-Santos, y Oliveira, 2001). Por otro lado, el debate con los profesores, que están en el inicio de su carrera y que fueron becarios del PIBID, puede fortalecer el desarrollo y/o fortalecimiento de políticas públicas dirigidas a las licenciaturas. 
Las reflexiones de los profesores participantes de esta investigación no dejan dudas en relación al papel de la iniciación en la docencia en la calidad de la construcción de los conocimientos profesionales. La oportunidad de proyectarse en el futuro espacio de profesión, posibilitada por el PIBID, contribuyó para la toma de consciencia sobre los contextos (faceta ecológica) en que irían, más tarde, a enseñar Matemática - una buena Matemática - (faceta epistémica). A lo largo de los momentos de planeamiento, ejecución y evaluación, los profesores articularon sus conocimientos, sea por el proprio repertorio de formación, sea por las reflexiones del grupo, aunque dispuestos a construir nuevos conocimientos.

Es esperado que profesores en inicio de carrera estén construyendo sus estrategias de actuación profesional y reelaborando sus conocimientos profesionales. Sin embargo, esa fase, dotada de complejidad, puede representar una oportunidad para que el profesor revele un equilibrio entre el campo del conocimiento matemático y el conocimiento didáctico (Silva, Pietropaolo y Font, 2016). Para tal, una formación inicial sólida y espacios de reflexión sobre la práctica son fundamentales, (Mena, Gómez, García, 2019). En ese punto, el PIBID marca mucha diferencia en el proceso de construcción de los conocimientos de un profesor de Matemática. Las revelaciones de los profesores sobre sus vivencias en el PIBID (inmersión en escuelas de educación, producción de informes sobre prácticas, socialización de experiencias en congresos y otros) comprueban su importancia y la necesidad de continuación como política permanente para la formación docente en Brasil.

\section{Referencias}

Abreu, I. S. M. de. (2016). Entre a singularidade e a complexidade da construção de saberes docentes na formação inicial de professores de matemática no contexto do PIBID. (Tesis de maestría). Universidad Federal de Goiás, Brasil. Recuperado en https://url.gratis/Qk2Qs

Amâncio, J. R. (2012). Planejamento e aplicação de uma sequência didática para o ensino de probabilidade no âmbito do PIBID. (Tesis de maestría) Universidad Federal de Rio de Janeiro, Brasil. Recuperado en https://url.gratis/cW12U

Ball, D. L., Lubienski, S. T. \& Mewborn, D. S. (2001). Research on teaching mathematics: The unsolved problem of teachers' mathematical knowledge. En V. Richardson (Ed.), Handbook of research on teaching (pp. 433-456). American Educational Research Association.

Ball, D. L., Thames, M. H. \& Phelps, G. (2008). Content knowledge for teaching. What makes it special? Journal of Teacher Education, 59(5), 389-407. 
Barros, A. V. (2016). Contribuições do Programa Institucional de Bolsas de Iniciação à Docência para a Formação Inicial dos Egressos das Licenciaturas da Universidad Federal de $A B C$. (Tesis de Maestría). Universidad Federal do ABC, Brasil. Recuperado en https://url.gratis/GzVWa

Benites V. C. (2013). Formação de professores de matemática: dimensões presentes na relação PIBID e comunidade de prática. (Tesis de Maestría). Universidad Estatal Paulista Júlio de Mesquita Filho, Brasil. Recuperado en https://url.gratis/dx7U6

Brasil, M. M. (2014). O PIBID no contexto das políticas de formação de professores de Biologia e Matemática na Universidad Estatal de Goiás. (Tesis de Maestría). Universidad Federal de Goiás, Brasil. Recuperado en https://url.gratis/DUSBf

Breda, A., Font, V. y Lima, V. M. R (2015). Propuestas de incorporación de contenidos matemáticos de nivel superior en la educación básica: un estudio de los trabajos finales de curso del Máster Profesional en Matemáticas en la Red Nacional. Revista Brasileira de Ensino de Ciência e Tecnologia, 8(3), 53-65. http://dx.doi.org/10.3895/rbect.v8n3.3189

Breda, A., Font, V. y Pino-Fan, L. (2018). Criterios valorativos y normativos en la Didáctica de las Matemáticas: el caso del constructo idoneidad didáctica. Bolema, 32(60), 255-278. http://dx.doi.org/10.1590/1980-4415v32n60a13

Canteiro, D. C. S. (2015). Impactos do Programa Institucional de Bolsa de Iniciação à Docência (PIBID) na formação inicial de professores de matemática. (Tesis de Maestría). Universidad de São Paulo, Brasil. Recuperado en https:/url.gratis/WGdTK

Carvalho, D. F. (2016). O PIBID e as relações com o saber, aprendizagem da docência e pesquisa: caracterização de uma intervenção na formação inicial de professores de matemática. (Tesis de Doctorado). Universidad Estatal de Londrina, Brasil. Recuperado en https://url.gratis/PXh32

Carvalho, M. P. (2016). Um estudo da inserção de estudantes da licenciatura em matemática no contexto da escola pública: contribuições do PIBID. (Tesis de Doctorado), Universidad Anhanguera de São Paulo, Brasil. Recuperado en https://url.gratis/Yj1J7

Corrêa, A. C. A. (2013). O PIBID na formação inicial do licenciando em Matemática: construção de saberes da experiência docente. (Tesis de Maestría). Instituto Federal de Espírito Santo, Brasil. Recuperado en https://url.gratis/PmY1J

Correia, G. S. (2012). Estudo dos conhecimentos evidenciados por alunos dos cursos de Licenciatura em Matemática e Física participantes do PIBID-PUC/SP. (Tesis de Maestría). Pontifícia Universidad Católica de São Paulo, Brasil. Recuperado en https://url.gratis/Ebyzd

Fiorentini, D. (2004). Pesquisar práticas colaborativas ou pesquisar colaborativamente? En Fiorentini, D y Araújo, J. L. (Eds.), Pesquisa qualitativa em Educação Matemática (pp. 4776). Autêntica. 
GARNICA, A. V. M. (2004). História Oral e educação Matemática. En Borba, M. C.; Araújo, J. L. (Eds.), Pesquisa Qualitativa em Educação Matemática (pp. 77-98). Autêntica.

Gatti, B. A. (2010). Pesquisa em Educação e Formação de Professores. En Teodora, R y Behrens, M. A. (Eds). Formação do Professor: profissionalidade, pesquisa e cultura escolar (pp. 117-134). Editora Universitária Champagnat.

Gatti, B. A., Barretto, E. S.S., André, M. E. D. A. y Almeida, P. C. A. (2019). Professores do Brasil: novos cenários de formação. Brasília, DF: Unesco.

Godino, J. D. (2009). Categorías de análisis de los conocimientos del profesor de matemáticas. UNIÓN, Revista Iberoamericana de Educación Matemática, 20, 13-31. Recuperado en https://url.gratis/fCzNB

Godino, J. D. (2011). Indicadores de la idoneidad didáctica de procesos de enseñanza y aprendizaje de las matemáticas. XIII CIAEM-IACME, Recife, Brasil. Recuperado en https://url.gratis/Ad7xw

Godino, J. D. (2013). Indicadores de la idoneidad didáctica de procesos de enseñanza y aprendizaje de las matemáticas. Cuadernos de Investigación y Formación en Educación Matemática, 11, 111-132. Recuperado en https://url.gratis/QMPbC

Godino, J. D., Batanero, C. y Font, V. (2008). Un enfoque ontosemiótico del conocimiento y la instrucción matemática. Acta Scientiae. 10, 7-37. Recuperado en https://url.gratis/2gRkg

Godino, J. D., Bencomo, D., Font, V. y Wilhemi, M. R. (2006). Análisis y Valoración de la Idoneidad Didáctica de Procesos de Estudio de las Matemáticas. Paradigma, 27(2), 221252. Recuperado en https://url.gratis/uoefh

Godino, J. D., Font, V., Wilhelmi, M. R. y Castro, C. de. (2009). Aproximación a la dimensión normativa en Didáctica de la Matemática desde un enfoque ontosemiótico. Enseñanza de las Ciencias, 27(1), 59-76. Recuperado en https://url.gratis/R1xC7

Godino, J.D., Contreras A. y Font, V. (2006). Análisis de procesos de instrucción basado en el enfoque ontológico-semiótico de la cognición matemática. Recherches en Didactique des Mathématiques, 26, 39-88. Recuperado en https://url.gratis/Lr1WC

Grossman, P. (1990). The making of a teacher: Teacher knowledge and teacher education. New York and London: Teachers College Press.

Hill, H. C., Ball, D. L., \& Schlling, S. G. (2008). Unpacking pedagogical content knowledge of students. Journal for Research in Mathematics Education, 39, 372-400.

Largo, V. (2013). O PIBID e as relações de saber na formação inicial de professores de matemática. (Tesis de Doctorado). Universidad Estatal de Londrina, Brasil. Recuperado en https://url.gratis/E5pY6 
Llinares, S.\&Krainer, K. (2006). Mathematics (student) teachers and teacher educators as learners. In A. Gutiérrez \& P. Boero (Eds.), Handbook of Research on the Psychology of Mathematics Education: Past, Present and Future (pp. 429-459). Rotterdam: Sense Publishers.

Mallart, A., Font, V. y Malaspina, U. (2015). Reflexión sobre el significado de qué es un buen problema en la formación inicial de maestros. Perfiles Educativos, 38(152), 14-30. Recuperado en https://url.gratis/oHfdi

Mena, J., Gómez, R. y García, M. L. (2019). La construcción de conocimiento pedagógico de los profesores en formación durante el prácticum. Revista Electrónica de Investigación Educativa, 21 y 27, 1-13. 10.24320/redie.2019.21. y 27.1831

Mendonça, S. R. P. (2016). Representação social sobre o ensino de matemática de licenciandos vinculados ao PIBID: dinâmica de formação. (Tesis de Doctorado). Universidad Federal de Rio Grande do Norte, Brasil. Recuperado en https://url.gratis/ZLDIJ

Mishra, P.\& Koehler, M. (2006). Technological Pedagogical Content Knowledge: A framework for teacher knowledge. Teachers College Record, v. 108, n.6, p. 1017-1054.

Moura, E. M. (2013). O programa institucional de bolsa de iniciação à docência - PIBID na formação inicial de professores de matemática. (Tesis de Maestría). Universidad Federal de Uberlândia, Brasil. Recuperado en https://url.gratis/zgnpG

Neves, R. M. S. (2014). Práticas de iniciação à docência: um estudo no PIBID/ IFPI/ Matemática. (Tesis de Maestría). Universidad del Rio de Los Sinos, Brasil. Recuperado en https://url.gratis/NKHxK

Palanch, W. B. L. y Manrique, A. L. (2016). Ações colaborativas universidade - escola: formação de professores que ensinam matemática em espaços colaborativos. Revista Eletrônica de Educação, 10,188-202. http://dx.doi.org/10.14244/198271991597

Pino-Fan, L. y Godino, J. (2015). Perspectiva ampliada del conocimiento didáctico-matemático del profesor. PARADIGMA, 36(1), 87-109. Recuperado en https://url.gratis/UnoNk

Pino-Fan, L., Font, V. \& Godino, J. D. (2013). Exploring the epistemic facet of the didacticmathematical knowledge required to teach the derivative. In Lindmeier, A.M. \& Heinze, A. (Eds.), Proceedings of the 37th Conference of the International Group for the Psychology of Mathematics Education(Vol. 5, p. 146). Kiel, Germany: PME. Recuperado en https://url.gratis/PnOdV

Pino-Fan, L., Font, V. y Godino, J. D. (2014). El conocimiento didáctico-matemático de los profesores: pautas y criterios para su evaluación y desarrollo. En C. Dolores, M. García, J. Hernández, y L. Sosa (Eds.), Matemática Educativa: La formación de profesores (pp. 137 - 151). México, D. F.: Ediciones D. D. S. y Universidad Autónoma de Guerrero. Recuperado en https://url.gratis/GdYlf 
Pino-Fan, L., Godino, J. D. y Font, V. (2013). Diseño y aplicación de un instrumento para explorar la faceta epistémica del conocimiento didáctico-matemático de futuros profesores sobre la derivada ( $1^{\text {a }}$ Parte). REVEMAT, 8(2), 1 - 49. http://dx.doi.org/10.5007/1981$\underline{1322.2013 \mathrm{v} 8 \mathrm{n} 2 \mathrm{p} 1}$

Pino-Fan, L., Assis, A., \& Castro, W. F. (2015). Towards a methodology for the characterization of teachers' didactic-mathematical knowledge. EURASIA Journal of Mathematics, Science \& Technology Education, 11(6), 1429-1456. https://doi.org/10.12973/eurasia.2015.1403a

Ponte, J. P., Galvão, C., Trigo-Santos, F.y Oliveira, H. (2001). O início da carreira profissional de professores de matemática e ciências. Revista de Educação, 10(1), 31-45. Recuperado en https://url.gratis/v0qH1

Ponte, J. P. \& Chapman, O. (2006). Mathematics teachers' knowledge and practices. In A. Gutierrez \& P. Boero (Eds.), Handbook of research on the psychology of mathematics education: Past, present and future (pp. 461-494). Rotterdam, The Netherlands: Sense Publishers.

Ponte, J. P. (2013). Aprendizagem em áreas de conhecimento: a matemática - Aprendizagem dos alunos e desenvolvimento profissional dos professores. In F. H. Veiga (Coord.), Psicologia da educação - Teoria, investigação e aplicação: envolvimento dos alunos na escola (pp. 333-358). Lisboa: Climepsi.

Ponte, J. P., Baptista, M., Velez, I. y Costa, E. (2012). Aprendizagens profissionais dos professores através dos estudos de aula. Perspectivas da Educação Matemática, 5, 7-24. Recuperado en https://url.gratis/nrT2g

Portaria Normativa No. 38. (2007). Regulamenta o Programa Institucional de Bolsa de Iniciação à Docência - PIBID. Ministério da Educação, Brasília, Brasil, 12 de dezembro de 2007. Recuperado de https://url.gratis/e1BSN

Ribeiro, S. S. (2012). Percepções de licenciandos sobre as contribuições do PIBID-matemática. (Tesis de Maestría). Universidad Federal de Lavras, Brasil. Recuperado en https://url.gratis/l1QQE

Ricoy, M-C y Couto, M. J. (2018). Desmotivación del alumnado de secundaria en la materia de matemáticas. Revista Electrónica de Investigación Educativa, 20(3), 69-79. https://doi.org/10.24320/redie.2018.20.3.1650

Rodrigues, M. U. (2016). Potencialidades do PIBID como espaço formativo para professores de matemática no Brasil. (Tesis de Doctorado). Universidad Estatal Paulista Júlio de Mesquita Filho, Brasil. Recuperado en https://url.gratis/mvYk9

Rowland, T., Huckstep, P., \& Thwaites, A. (2005). Elementary teachers' mathematics subject knowledge: The knowledge quartet and the case of Naomi. Journal of Mathematics Teacher Education, 8(3), 255-281. 
Schaefer, C. (2015). Experiências e narrativas: um olhar para a formação de professores de matemática a partir do PIBID. (Tesis de Maestría). Universidad de Santa Cruz do Sul, Brasil. Recuperado en https://url.gratis/1jRE1

Schoenfeld, A., \& Kilpatrick, J. (2008). Towards a theory of profiency in teaching mathematics. En D. Tirosh, \& T. L. Wood (Eds.), Tools and processes in mathematics teacher education (pp. 321-354) Rotterdam: Sense Publishers.

Shulman, L. S. (1986). Those who understand: Knowledge growth in teaching. Educational Researcher, 15(2), 4 - 14.

Shulman, L. S. (1987). Knowledge and teaching: Foundations of the new reform. Harvard Educational Review, 57(1), 1-22.

Silva, E. C. (2014). Ações e reflexões de licenciandos sobre o ensino-aprendizagem da álgebra no PIBID-Ifes. (Tesis de Maestría). Instituto Federal de Espírito Santo, Brasil. Recuperado en https://url.gratis/5ZEVM

Silva, J. F.; Pietropaolo, R. C. y Font, V. (2016). Relações entre conhecimentos e competências na formação inicial de professores de matemática. Acta Latinoamericana de Matematica educativa, 28,861-867.

Tinti, D.S. (2012). PIBID: um estudo sobre as suas contribuições para o processo formativo de alunos de Licenciatura em Matemática da PUC-SP. (Tesis de Maestría). Pontificia Universidad Católica de São Paulo, Brasil. Recuperado en https://url.gratis/1rCO5

Tinti, D.S. y Manrique, A. L. (2019). PIBID: Inserción de futuros profesores de matemáticas en el entorno escolar con vistas a minimizar el choque con la realidad. Linhas Críticas, 25, 333349. Recuperado en https://url.gratis/mcxEE

Tinti, D.S. y Manrique, A. L. (2016).Teoria e Prática na Formação de Professores que ensinam Matemática: que caminhos apontam experiências com o PIBID e OBEDUC?. Educação Matemática em Revista (São Paulo), 49, 98-106.

Vicente, M. F. (2016). Programa institucional de bolsas de iniciação à docência- PIBID - e a formação inicial de professores. (Tesis de Maestría). Universidad Estatal Paulista Júlio de Mesquita Filho, Brasil. Recuperado en https://url.gratis/pu6ns

Vieira, A. C. (2014). Um estudo sobre as contribuições do PIBID-FURB para a formação inicial de professores de matemática. (Tesis de Maestría). Universidad Regional de Blumenau, Brasil. Recuperado en https://url.gratis/3E0sZ

Zaqueu, A. C. M. (2014). O Programa Institucional de Bolsas de Iniciação à Docência (Pibid) na formação de professores de matemática: perspectivas de ex-bolsistas. (Tesis de Maestría), Universidad Estatal Paulista Júlio de Mesquita Filho, Brasil. Recuperado en $\underline{\text { https://url.gratis/qHQz0 }}$ 
José Fernandes da Silva \& Ana Lúcia Manrique 